\title{
Challenges and Potential Solutions for $100 \%$ Recycling of Medical Textiles
}

\author{
Sourav Kumar Das ${ }^{1} \cdot$ Amutha Chinnappan $^{2}$ (D) W. A. D. M. Jayathilaka ${ }^{2} \cdot$ Rituparna Gosh $^{2} \cdot$ Chinnappan Baskar $^{3,4}$. \\ Seeram Ramakrishna ${ }^{2}$ (D)
}

Received: 26 January 2021 / Revised: 4 May 2021 / Accepted: 7 May 2021 / Published online: 20 July 2021

(c) The Author(s), under exclusive licence to Springer Nature Singapore Pte Ltd. 2021

\begin{abstract}
Medical textiles are all fiber-based products and structures which are utilized for emergency treatment, clinical, surgical and hygienic purposes. It is an exceptionally particular and bio viable specialized material, utilized for clinical and cleanliness applications. Volumes of clinical waste being created in excess of 60 million tons yearly around the world. According to the current investigation reports and information, the worldwide clinical waste administration market was esteemed at USD 11.77 billion in the year 2018 and will reach at 17.89 billion by the year 2026 at a compound annual growth rate (CAGR) of $5.3 \%$. Over the world, out of the measure of waste created by medical care activities, about $85 \%$ is general waste and staying $15 \%$ is viewed as unsafe material that might be irresistible, poisonous or radioactive. The following particular reasons are very harmful for the environment in the upcoming future. The waste management policy of medical textile is a vital fact for the world. The potential and effectual solution is recycling of these medical wastes. Current solutions for $100 \%$ recycling of medical textiles are chemical treatment, incineration, and autoclaving. But the most innovative solution of medical textiles is molecular tagging/tagging of fibers. Medical textile market is producing state-of-the-art polymeric textile implantable devices that are redefining traditional materials and methods of surgery. Developing polymer innovation has yielded a wide scope of uses of implantable clinical material or biotextiles. Due to world Covid-19 pandemic situation, the requirement of medical textiles already has been increased almost double from last year. It has been observed that the market value of medical textiles will be in optimum position. In the year 2019, the global market worth of medical textiles was US\$ 17.5 billion. In the present world, the current medical textiles like implantable and non-implantable categories are not applying for recycling process or end used of their life cycle. In this paper, we will discuss about potential solutions for recycling medical textiles like—by using conductive polymers, maintaining $\varepsilon$-Poly-lysine, non-fibrous biomass, bioactive fibers, etc. But there are still some challenges for recycling like-maintain $100 \%$ polymeric bonds, bacterial effect, flexibility and sustainability for clinical performance after recycling the specific product. In this paper, we are presenting the scientific methods, mechanisms, and procedures that used to overcome the aforementioned challenges in the recycling methods.
\end{abstract}

Keywords Recycle $\cdot$ Challenges $\cdot$ Solutions $\cdot$ Medical textiles $\cdot$ Anti-viral $\cdot$ Nanoparticles and polymers

Amutha Chinnappan

mpecam@nus.edu.sg

$\triangle$ Seeram Ramakrishna

seeram@nus.edu.sg

1 Wuhan Textile University, Wuhan, China

2 Center for Nanotechnology and Sustainability, Department of Mechanical Engineering, National University of Singapore, Singapore 119260, Singapore

3 THDC Institute of Hydropower Engineering and Technology Tehri, Uttarakhand Technical University, Dehradun, India

4 Faculty of Mathematics and Natural Sciences, Pattimura University, Ambon, Indonesia

\section{Introduction}

Over the few years, the medical textile industry is developing with new techniques for obtaining the combination of clinical purposes and optimization. The rate of successive challenges of recycling medical textiles is growing day by day with a significant result and flexible performance. With such widespread interest emerging in almost every area of daily life in the recycling and re-use of medical textile products, healthcare is one that does not immediately come to mind. There are, however, millions of textile products used per year in the healthcare sector. Many items become 
contaminated with medications, bodily fluids and blood during use, which makes it impossible to recycle or reuse. Because of strict restrictions on interaction with body fluids and medical devices, there is a growing demand for disposable products in health care. Most textile products placed on the healthcare market are currently incinerated or sent to landfill, with virtually all disposable products incinerated after use (Salahudeen and Rasheed 2020).Three main classes of antiviral coatings (antiviral polymers, metal ions/ metal oxides, and functional nanomaterials) were divided on the basis of the form of materials used at the contaminated sites. Based on the form of materials used at the virus contamination sites, the constituent antiviral members are categorized into a few large categories, such as polymeric materials, metal ions/metal oxides, and functional nanomaterials. To vindicate the antiviral process, the action mode against enveloped viruses was depicted. In determining, for example, between recycling potentially polluted goods and disposing of the goods in landfills, industry must take local environmental factors into account. However, another approach to this issue should be used, taking into account the global effect of these products on the environment, such as the analysis of the product life cycle (Abreu et al. 2006). In the high tech textiles and apparel industry, medical textiles (medtech) are considered one of the main growth areas. The advent of new and improved raw materials and processes is clearly motivated by Medtech, leading to new technical innovations explicitly designed to tackle issues facing medical professionals and patients on a daily basis (Sungmee and Jayaraman 2005).

Human discharge about 1.5 million tons of essential microplastics in to the sea annually, an aggregate identical to one ejected plastic sack for each individual each week. Such contaminations presently make up as much as $31 \%$ of all sea plastic and they turned up all over marine environment (Karim et al. 2020). A far reaching concentrate in the year 2017 found an issue that only $2 \%$ of such plastics originate from microbreads which a few nations have begun restricting. The driving source - at 35\% ended up being materials produced using engineered textiles (Ahmed et al. 2016). In second spot, at $28 \%$ were the scraped pieces of vehicle and truck tires that wash from streets into waterways (Wicaksono et al. 2020). British purchasers are now requesting supportable items and shopping experiences. In time, they will anticipate that retailers and brands should address the items that are really adding to the problem. The uplifting news is that few organizations are in good shape. Leading clothing brands - including Patagonia, Adidas, and Mauritz - have recognized that their manufactured pieces of clothing are a significant wellspring of plastic microfiber contamination (Grasso 1995).

For certain enterprises, recycling has, for many years, been a mode of service. The various staple lengths and degree of maturity in the fibers are a major problem with recycled waste fiber. These characteristics make the recycled yarn ideal for a small range of products, such as mop heads. Jones Companies Ltd., since 1936, has been recycling waste fiber and has gradually improved the quality of yarn. The organization is concerned with manufacturing better quality yarn so that they can manufacture goods of higher quality. The growing issue of infectious disease worldwide, previously referred to, actually has a detrimental effect on the overall population around the Earth. More specifically, the problem presents a direct threat to the healthcare staff of our society, responsible for treating the sick. The World Health Organization (WHO) has estimated that there are some 59 million healthcare staff in the six regions of the world they have listed. Almost all of these individuals are threatened, to some degree, by infectious diseases, depending on their particular responsibilities (Zins et al. 2015). Due to their ability to incorporate textile-based sensing and monitoring technologies into everyday life, functional textiles are seen as promising technologies to allow healthcare facilities and medical care outside hospitals. Much work has already been spent on fundamental practical textile research in the past, demonstrating that reliable monitoring solutions can be realized. The task remains to define and improve suitable medical applications and to meet the boundary criteria for medical approval and exploitation (Reiter et al. 2011). A fabric's key benefit is its ability to adapt to shape and act as a "platform" or infrastructure to "carry" sensors and other information processing devices (from small to large) over various surface areas. There is no contact between the fabric and the electronic elements in such a loosely coupled mode, and the information carrying capabilities of textile fibers are not harnessed (Rajendran et al. 2002).

In recent years, antimicrobial textiles have drawn a significant number of research activities aimed at reducing the spread of infectious diseases and enhancing the quality of life of human beings. In terms of killing strength, speed and effectiveness, textile materials for medical applications have unique antimicrobial performance specifications against a variety of microorganisms, such as bacteria, viruses and even spores that are somewhat different from the needs of consumer goods. When applied to fabrics and fibers, various biocides with claimed antimicrobial functions against pathogens are also available (Sun et al. 2011). The practice of recycling textile waste is prevalent in many countries, such as the UK, Germany, the USA, and Australia. Using passes, vouchers, competitions, and payments as lottery prizes to collect public residential engagement in recycling schemes, initiatives have been used to boost positive behavior toward recycling in schools (Ahmed et al. 2016).

Because of the widespread, medical textiles account for a huge market, not only in the hospital, hygiene and healthcare sectors, but also in hotels and other environments 
where hygiene is required. The use of natural as well as synthetic fibers in the manufacture of different medical products has increased dramatically. During 1999-2000, the annual growth of medical textile products was projected to be about 10\% (Rajendran et al. 2002). Personal protective equipment (PPE) is important for the safety of healthcare workers (HCWs) from highly infectious diseases such as Covid-19. However, due to unprecedented global demand, hospitals have been at risk of running out of the safe and efficient PPE, including personal protective clothing needed to treat patients with Covid-19. Moreover, due to the lack of available knowledge of related technology, inadequate supply chains and strict regulatory requirements (McCullough et al. 1995), there are only limited production facilities for such clothing available worldwide. There is, therefore, a strong unmet need to coordinate the activities and efforts of scientists, engineers, manufacturers, suppliers and regulatory bodies to develop and manufacture safe and efficient protective clothing using technologies available locally around the world (Karim et al. 2020). There are current comparisons of reusable and single-use perioperative textiles (surgical gowns and drapes). For medical staff and patient safety, reusable and disposable gowns and drapes follow new requirements, use synthetic lightweight fabrics, and are competitively priced (Overcash 2012).. Reusable surgical gowns and drapes show major sustainability advantages in many science-based life cycle environmental studies over the same disposable commodity in natural resource energy, hazardous organics, solid waste and instrument recovery (Brasch et al. 2013). Soft fibers and functional fabrics become an ideal platform in the areas of biological and health monitoring, implants and implants, movement implants, artificial intelligence, and human machine communication. A major challenge to their sustainable performance lies in the need for reliable and reputable energy systems (Dong et al. 2020). In the recent years, scientists have also focused on the profusion of so-called micro process, which are about the size of a sesame seed or less. Some of these are generally regenerated by the breakdown or abrasion of the larger products. In the last couple of years, the medical textiles waste like billions of tones are hampering our environment seriously as well as it is a big chance to reuse by recycling process for playing a new horizon of the economy. By this review, we did our outmost to focus this serious matter with this recent and current incident of medical textile recycling process. Nowadays, we can observe that the whole world is struggling with Covid-19 pandemic situation with lack of sufficient medical aids. With this review and research paper, we can implement a great contribution on behalf of medical textiles recycling process with proper technology so that the medical aids will be sufficient and have a great contribution in the world in terms of economy, pandemic situation, insufficient supplies.

\section{Materials}

For medical and surgical uses, various materials are used. Closely woven cotton is commonly used in operating theaters around the world and has some capacity to resist infection transmission by direct contact when dry. It has no resistance to wetting out, however, even when fresh. Once it becomes wet, cotton barrier performance is impaired, no matter how many layers are used. Consequently, such cotton fabrics are not appropriate for the operating theater. Thus, the search for a material immune to aqueous penetration started and a new generation of materials started. A nonwoven fluid-resistant fabric designed for use only once and discarded, as well as a variety of modern, tightly woven, reusable non-absorbent fabrics, are among them. In long, complex procedures where a high incidence of liquid and high-pressure mechanical stress related behavior occurs, laminates ensure reliable safety. Sustainability is one of the key challenges of modern society and in this regard, the science of building materials has evolved into the installation of highly polluting substances that have a low impact on the environment. Polymeric materials are no different. It is estimated that the production of plastics worldwide is about 320 million tons annually and is increasing and only about $10 \%$ of that amount is recycled, which means a large generation of waste and its dramatic effects on the global environment (He et al. 2016) to be used in new applications within areas such as food preparation. Cellulose is probably the most interesting of these biopolymers because it is so abundant on Earth and for centuries has had a profound impact on technology in areas such as textiles, packaging and storage. Cellulose is a polysaccharide caused by duplication of glucose units (Salahudeen and Rasheed 2020).

Performance comparisons between before and after recycled products were confirmed after a review, the performance of recycled medical textiles were significantly indicated in present days (Zhao et al. 2009). Reusable thermostat water is supplied with bath water means thermo. Using this application, the maximum reaction temperature was $100^{\circ} \mathrm{C}$ and temperature fluctuations could not be controlled below low or medium microwave power and this thermal technology can be used in medical textiles recycling process (Mora et al. 2019).

Liquid blending of liquid or liquid chemicals is widely used in petrochemical processes, textile printing, food and the medical industry. These complex fluids must usually be separated after reaction for the purpose of product purification, regeneration or safe discharge (Hou et al. 2016). Another group of natural peptide products 
Fig. 1 Global life-cycle green house gas (GHG) emissions of conventional plastics in 2019 by life-cycle stage and recycle type. Blue, orange, and green management, conversion and EoL management, respectively. The discharge of each phase is classified by the type of recycling treatment method, indicated by different colors of the corresponding color represent the stages of resin

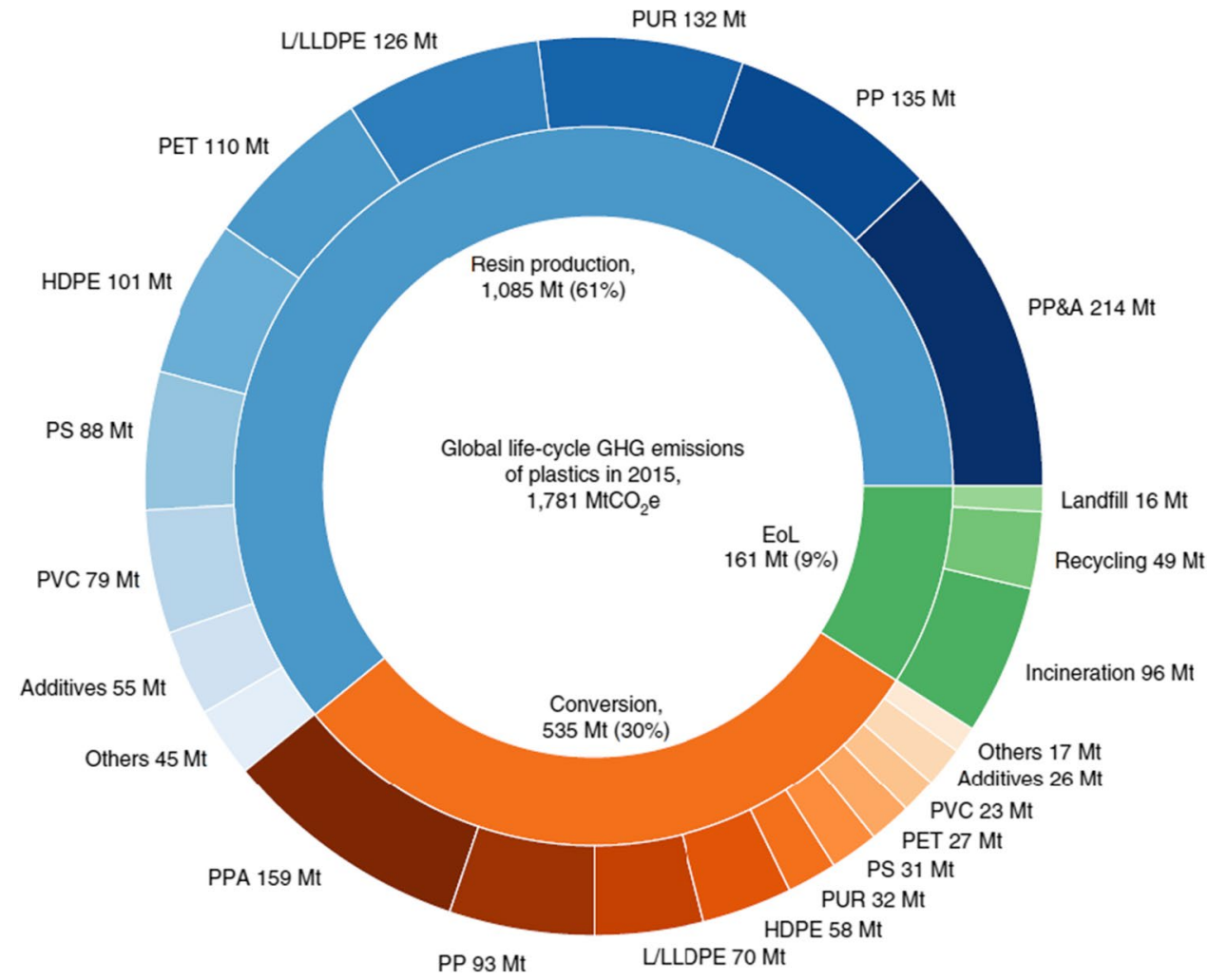

produced by sophisticated molecular machines is ribosomally synthesized and post-translation-modified Peptides (Karim et al. 2020). Insoluble melt pollution. Such industrial processes include plastics, leather, paint, fabric, acrylic, etc. (Salahudeen and Rasheed 2020). Low carbon power is another strategy to reduce the release of plastic GHG life cycle. Under the condition of $100 \%$ renewable energy, GHG emissions from US plastics production can be reduced by 50-75\%. Another strategy to reduce GHG emissions from plastic is recycling, which reduces, in part, the more potent virgin production while preventing GHG emissions from other endogenous processes (EoL) such as incineration (Zheng and Suh 2019) (Fig. 1).

The pore size is customized so that the membrane cannot be breached by bacteria, viruses and liquids, but the passage of water vapor molecules is possible. At high pressures, the laminate must be liquid-tight and show greater liquid resistance than other materials which are liquid-repellent. A barrier is something that hinders or limits by definition; (Brasch 2014) it may be something that hinders entry or passage; or it may be something that prevents or delays passage. Fabric barrier properties can, therefore, be characterized as the ability of a material to resist the penetration of liquids and/or micro-organisms, referred to as 'strike-through'. We can retain 3R technology for recycling materials in this phase (Fig. 2). (a)
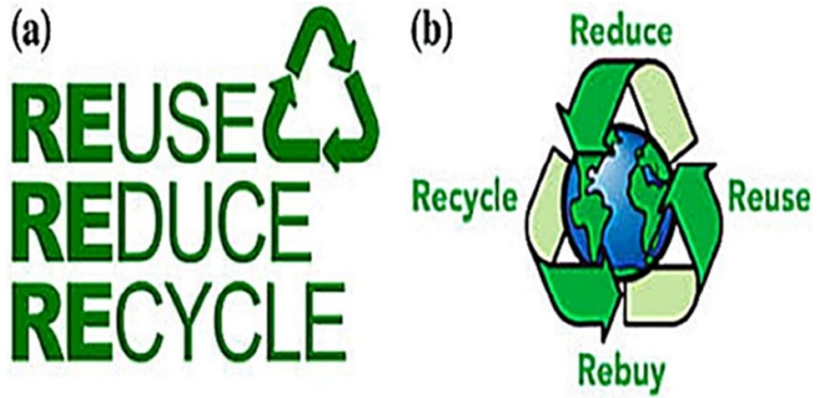

Fig. 2 Concepts of $3 \mathrm{R}$ in recycling materials

\section{Molecular level tagging of polymers in fibers}

In medical applications fields such as anti-counterfeiting, biological mimicking, data storage, sequence-defined macromolecules consist of a defined polymeric chain length, end groups, composition and topology. We have tried here the potential use of a storage medium of multifunctional sequence related macromolecules so that the performance after recycling will remain good durability (Rucker et al. 2011; Samanta et al. 2015). Although current traditional storage devices are still able to cope up with a chain of polymeric functional stability, polymeric molecular tagging densities can be significantly increased and element sources $(\mathrm{C}, \mathrm{H}, \mathrm{N}, \mathrm{O})$ are highly abundant 
as components of macromolecular information. DNA, the genetic information carrier and potentially the largest biopolymer of nature has already been used as a macromolecule (Shimizu et al. 2020; Sun 2020; Swift 2020; Takamatsu et al. 2020). We need fundamentally denser storage technologies to store and make use of the traditional knowledge (Carmean et al. 2019) to give proper stability of moleculars tagging chains. Historically, two distinct disciplines have separately studied biological and man-made polymers. The analytical tools developed for the characterization of bio-macromolecules are, therefore, often very different from those used for synthetic polymer analysis. The size of biopolymers, for example-is classically measured (Zins and Ellas 2007) by gel electrophoresis, while synthetic polymers are more generally analyzed by gel permeation chromatography. However, as modern synthetic polymers have recently become almost as structurally complex as biopolymers, the historical and cultural barriers between disciplines are increasingly disappearing. Not only is DNA's ability to store and process information important for biology, but it also suggests a possible new path for man-made information technology (Tebyetekerwa et al. 2020; Traka et al. 2019; Vadicherla and Saravanan 2019). For example, it has been stated that a whole book can be stored in the form of DNA sequences. Obviously, this biopolymer is very desirable for use in initial information of storage applications because there are already available methods of synthesizing, copying and reading it, built and honed through decades of biomolecular study (Colquhoun and Lutz 2014) (Fig. 3).

ESI mass spectrometry analysis was performed on a Synapt G1 HDMS mass spectrometer (Waters). Tested samples for this above polymeric reactions were diluted in 50\% acetonitrile/ $0.1 \%$ formic acid in water and transferred into a 96-well plate (Martens et al. 2018) (Fig. 4).

In living organisms, three sequence-controlled polymerization processes are dominant: replication of DNA, Transcription of DNA-RNA, and translation of RNA-protein, in which the molecular information carried by nucleic acids is translated into protein chains identified by sequence. Nucleic acid-templated polymerization takes place during DNA replication and transcription through the action of DNA and RNA (Zhang et al. 2019). The mechanism of translation is much more complex and relies on large catalytic particles consisting of both RNA and proteins from ribosomes. All three of these polymerizations are more closely regulated than any known processes of synthetic polymerization. Therefore, biological methods for polymer synthesis and materials science have clear advantages (Mutlu and Lutz 2014).

When the stability drawbacks of the biocatalyst in unnatural environments (i.e., organic solvents, high temperatures) are prevented through the use of immobilization-stabilization techniques, the efficiency of enzymes in transforming substrates is also enhanced (Zhao et al. 2019). Genetic mapping offers an important method at the molecular level to understand the genetic architecture of quantitative traits (Zins and Howard 2011). Thousands of QTLs responsible for lint yield, yield elements, fiber quality and other traits have been mapped and characterized using $\mathrm{G}$ with the construction of high density genetic maps of tetraploid cotton, respective standards-the-G. Inter-specific populations of F2, BC and RIL in Barbadense (Zarybnicka et al. 2017).In the textile industry, fiber is a fundamental raw material.). In this analysis, the QTLs detected provide new knowledge to improve the quality of fiber and especially useful for markerassisted selection (Zhiyuan et al. 2014).
Fig. 3 Self-immolative antibacterial polymers spontaneously unzip into small molecules in response to a specific chemical stimulus (poly(benzyl ether)s with pendant allyl side chains and silyl ether end-caps) (Ergene et al. 2018)

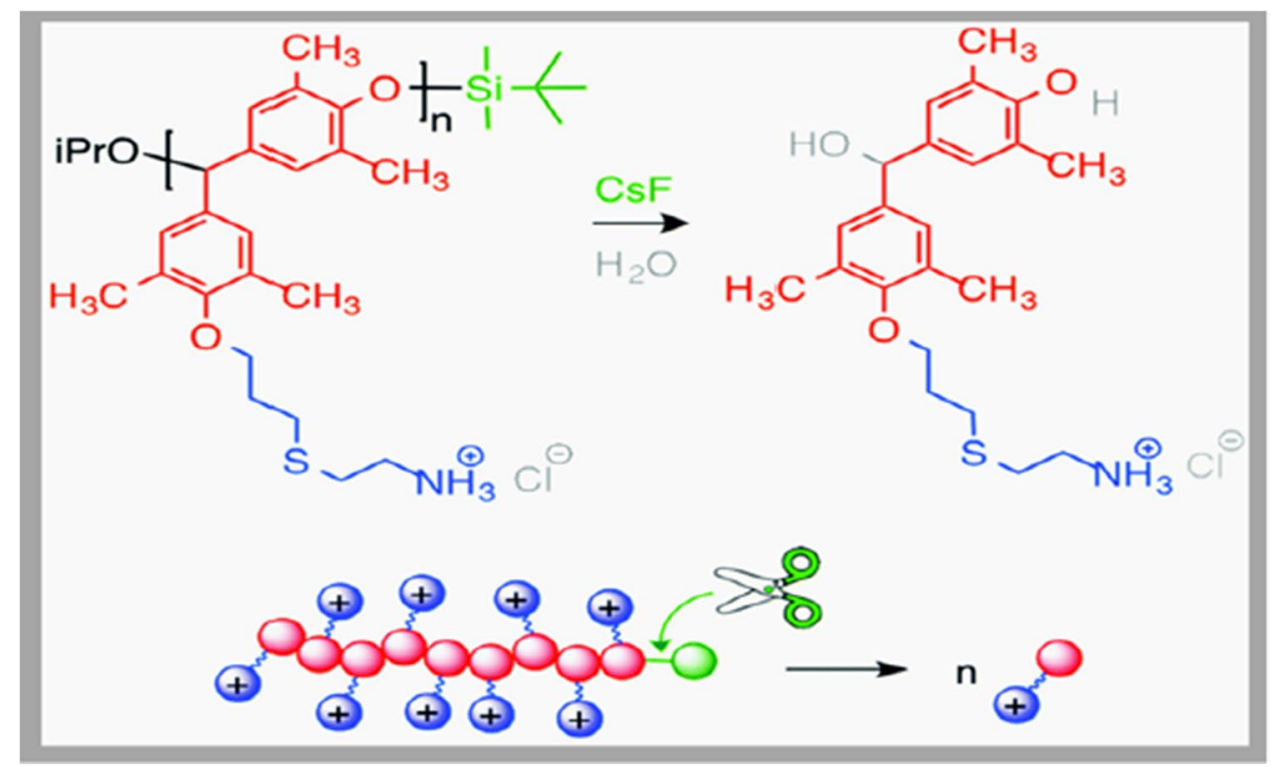



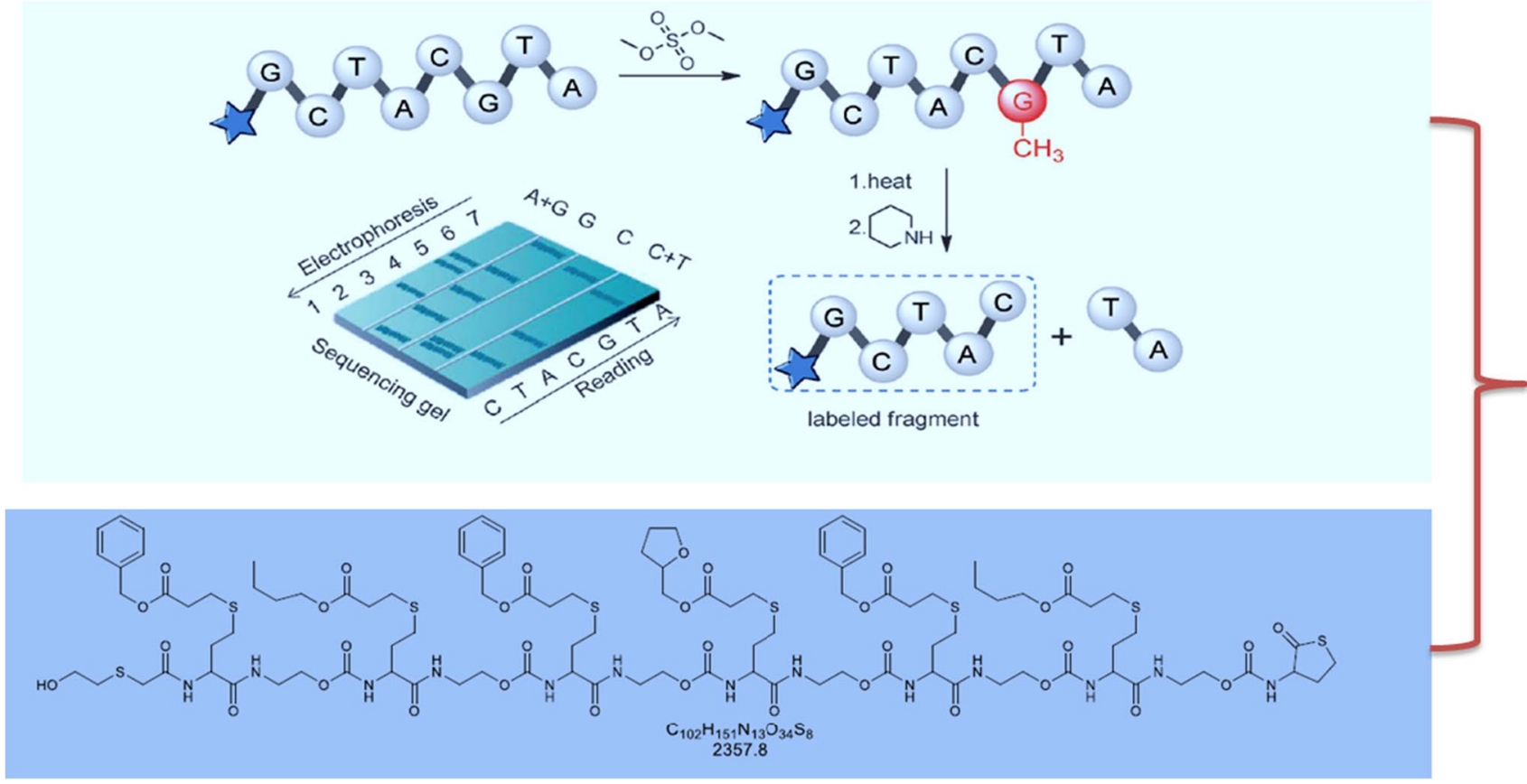

Fig. 4 Identification of a sequence in batch polymers $\left(\mathrm{C}_{102} \mathrm{H}_{151} \mathrm{~N}_{13} \mathrm{O}_{34} \mathrm{~S}_{8}\right)$ with tagging via mass spectroscopy (Algorithm determines the composition of a sequence-defined polymer starting from an experimental MS/MS-spectrum of the polymer (Wicaksono et al. 2020)

\section{Challenges}

After recycling done on specific materials used for medical textiles, there are some challenges for reuse with $100 \%$ comfortable way and full flexibility.

\section{Critical incident occurrence for surgical applications}

We can see that most recycled items are similar to product failure or critical incidents can be used as a way to direct product creation during product use for surgical gowns and drapes, however in this research, critical incidents were used to assess if one-shot learning occurred when one form of product had a major problem (McCullough 1993; Moradali and Rehm 2020; Moradi et al. 2019; Mutlu and Lutz 2019). The Critical Incident Technique (CIT) involves asking respondents to define specific cases where products are particularly efficient or ineffective. The events are then classified into groups and reliability checked (Brasch et al. 2015).

\section{Resistance to microbial penetration}

With large quantities of body fluids involved, some operations are very invasive and others are considered to be very high risk, such as orthopedic and neurosurgery. Others are considered to be low risk. The end users are responsible for deciding what fabrics they want to use and for which surgical procedures they want to use. The consumer acknowledges the duty not to use fabrics for which they were never intended (Newsome et al. 1993; Nezakati et al. 2018; Payne 2019).

The clarity of the thin and soft fibers lies in the fact that they can be woven inside fabrics using a variety of welldesigned techniques such as weaving and knitting (Pensupa et al. 2018; Radu et al. 2016). The most powerful and sensitive respiratory monitoring program we have shown is designed to detect early signs of respiratory distress associated with various medical conditions in sleep pathologies or severe viral symptoms (Wicaksono et al. 2020).

The conveyors need to be installed not only on the surface of the woven structure but also on the inside, providing continuous contact between the wires during its mechanical deformity (Takamatsu et al. 2015). From a molecular standpoint, sulfobetaine is closely related to taurine (2-aminoethane sulfonic acid), which contributes significantly to human and animal tissue cells (Rajan et al. 2020; Rajendran and Anand 2018). Because of these benefits, sulfobetaines are highly promising as excellent constituents in the earth's performance in textile materials. They have not only provided antimicrobial properties in textile care and wound dressing, but also improved textile hydrophilicity, which, however, has very few reported sulfobetaine active compounds in textile materials (He et al. 2016) (Table 1).

In this above table with mentioned polymers, there have been some disadvantages for this replacement. This is given below: 


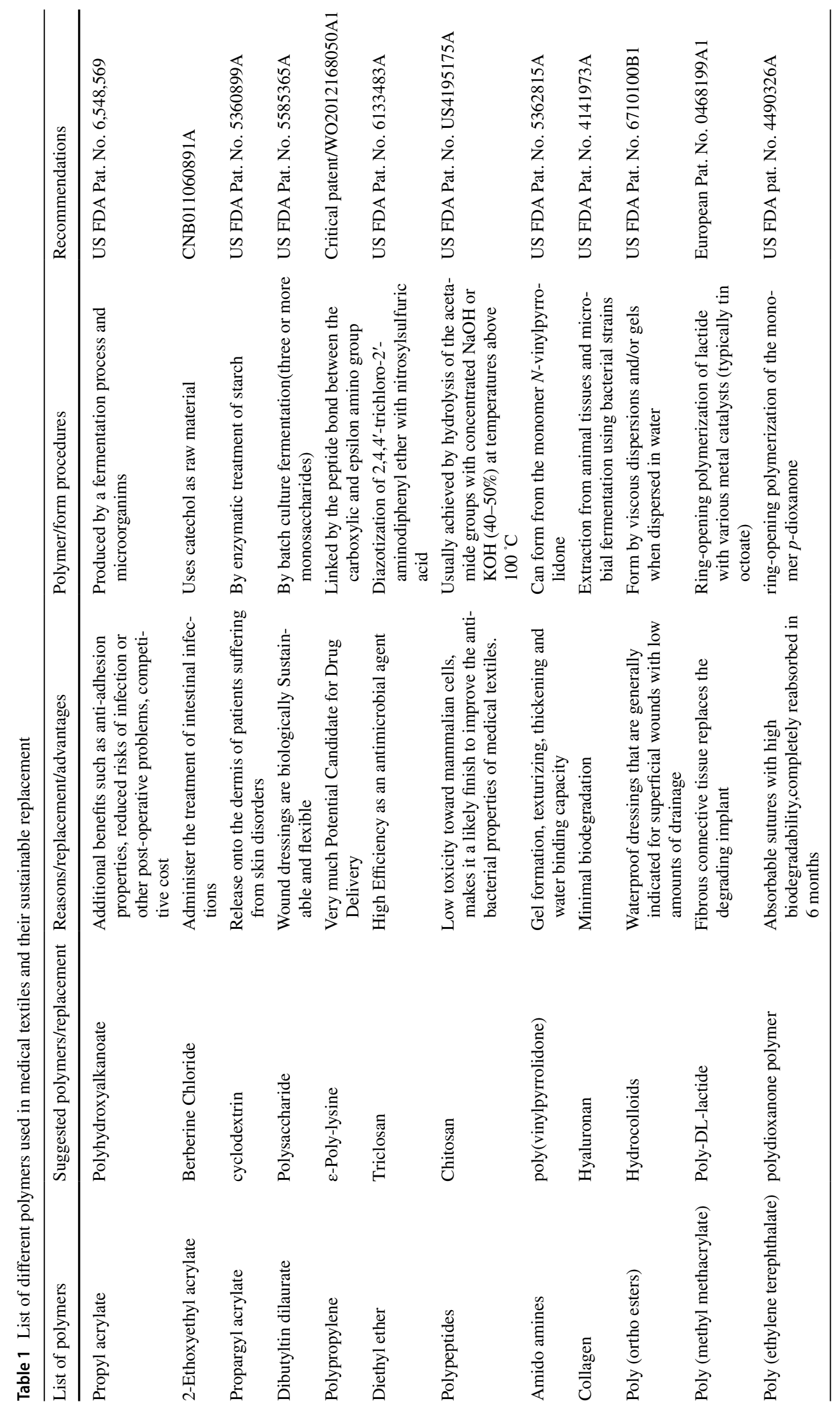


- Polyhydroxyalkanoate- poor mechanical properties, high production cost, limited functionalities

- Berberine Chloride- some less side effect for children.

- cyclodextrin- Penicillin derivatives are sensitized

- Polysaccharide- enhancement of pharmacokinetic properties

- $\varepsilon$-Poly-lysine - can produce L-lysine through polymerization

- Triclosan- Alters hormone regulation in animals.

- Chitosan- impacts on alkaline waste and organic material.

- $\operatorname{poly}$ (vinylpyrrolidone) - slowly added to ethanol with vigorous

- Hyaluronan- can cause redness and soreness when injected into the joint.

- Poly-DL-lactide- attempts to address the effects of PLGA molecular weight, lactide to glycolide ratio, crystallinity

- polydioxanone polymer- $7.9 \%$ rate of delayed noninfectious inflammatory tissue response requiring surgical drainage

\section{Resistance to liquid penetration}

There is a need to maintain the traceability of decontamination, disinfection and sterilization. Using an agreed manufacturing or other methods that can preserve the properties of the material, the properties of the materials will have to be preserved (Ahmad et al. 2019). A manufacturing specification, including visual and hygienic cleanliness, decontamination, disinfection and sterilization, would have to be developed and tested for the product. A product that has been successfully designed could be transformed into an unrecognizable piece of material after a sterilization method (Arun and Gopinath 2020).

\section{Bursting strength}

Sustainability is the world's most recent buzzword that encompasses three areas, including the environment, economics and culture (Brasch et al. 2019). With the advent, acceptance, and obsolescence of quick fashion, textile waste is rising at phenomenal rates to unparalleled levels. Via a proper recycling approach, textile waste can be the raw material for the production of new value-added goods (Brennan 2018). Currently, textile waste management systems are undergoing a critical process aimed at generating value-added goods through different concepts and methods of recycling (Lukasz et al. 2019).

\section{Tensile strength}

Basically, mechanical recycling is a melt extrusion process consisting of the removal of contaminants and the melting of polymer flakes. Sorted polyester waste is ground into flakes and washed to eliminate the pollutants with detergent or solvents (Colquhoun and Lutz 2021). Flakes are thoroughly dried to avoid contamination of the products by hydrolytic degradation. To improve mechanical characteristics, these flakes are melted, extruded, and ideally cross-linked (Edwards and Vigo 2001). For a wide variety of items which would otherwise be made of virgin polyester fibers, such as yarns and other items, PET flakes are used as the raw material (Karim et al. 2020).

\section{Lint generation}

During surgical procedures, airborne particles, specifically lint, may cause serious complications. Nonwoven fabrics made from wood pulp fiber, for example (He et al. 2019). Among other items, peritoneal adhesions have been reported as contributing to post-surgical difficulties such as intestinal obstructions (Sharfman et al. 2001). Incisional hernias and chronic abscesses at the wound site were additional postoperative problems (Das et al. 2020).

\section{Potential solutions}

The innovation solution for recycling of medical textiles is molecular tagging. However, we are discussing some other process for these solutions.

\section{Incineration}

The burning of natural mixes creates chiefly vaporous emissions, including steam, carbon dioxide, nitrogen oxides, and certain toxic substances (for example, metals, halogenic acids), and particulate issue, plus solid deposits as remains (Hou et al. 2019). In the event that the states of burning are not properly controlled, poisonous carbon monoxide will likewise be created. The wastewater delivered by the cycle likewise contain harmful compounds, which must be blessed to receive keep away from antagonistic impacts on wellbeing and the environment (Hug et al. 2020). Most huge, present day incinerators incorporate energy-recuperation offices. In cold atmospheres, steam and additionally high temp water from incinerators can be utilized to feed metropolitan region warming frameworks, and in hotter atmospheres, the steam from incinerators is utilized to create power (Kozlowski et al. 2010). 


\section{Ethylene oxide treatment}

Ethylene oxide treatment is a setup innovation, having been utilized for quite a long time to sanitize materials - items available to be purchased, squander for removal, and things for reuse (Kumartasli and Avinc 2020). In clinical offices, ethylene oxide is utilized to treat catheters, stents, hardware with coordinated gadgets, and different articles that cannot withstand the high temperatures of autoclaves. Surgical blades, cinches, other metal items and be treated with EtO despite the fact that they are all the more normally disinfected in autoclaves (Leung et al. 2020). Ethylene oxide, generally truncated $\mathrm{EtO}$, which has the compound equation $\mathrm{C} 2 \mathrm{H} 4 \mathrm{O}$, is once in a while called oxirane. It is a risky synthetic, however, it has qualities that make it ideal for clump cleansing of odd-formed items. Ethylene oxide is a gas (unadulterated EtO bubbles at around $50^{\circ} \mathrm{F}$. It is regularly blended in with a transporter gas, for example, nitrogen. It can go through plastic sacks and other penetrable bundling. In an all around planned cycle, the EtO encompasses the waste and purifies the surfaces. EtO pointer tape (much like the marker tape utilized in autoclaves) shows administrators that the sanitization cycle has been finished (Ma et al. 2020).

From a molecular standpoint, sulfobetaine is closely related to taurine (2-aminoethane sulfonic acid), which contributes significantly to human and animal tissue cells. Because of these benefits, sulfobetaines are highly promising as excellent constituents in the earth's performance in textile materials (Mayandi et al. 2020). They have not only provided antimicrobial properties in textile care and wound dressing, but also improved textile hydrophilicity, which, however, has very few reported sulfobetaine active compounds in textile materials (He et al. 2016).

\section{Containing $\varepsilon$-Poly-lysine fixation}

In terms of surface area, skin is the human body's largest organ, with a multitude of functions, including shielding our body from external pathogens. This barrier function is impaired by any sort of injury or impairment to the skin. A cascade of simultaneous stages, known as wound healing, involves effective cutaneous repair. Burn patients have a high risk of infection, caused by both resident and nosocomial acquired infections, which leads to a higher rate of mortality. Contemporary burn injury treatment requires both the patient's survival and the burn tissue's functional and cosmetic result. Electrospinning is a flexible tool for the development of tissue engineering and regenerative medicine nanofiber scaffolds. The evolution of multi-drug resistant pathogens during hospital stay after burn injury has been shown by a number of single-centered studies (Edwards et al. 2010).

\section{Breathable fluid barrier}

A significant research area has always been the production of suitable textile materials for the safety of healthcare workers in the operating rooms. Tri-layer fabrics for surgical gown applications have been produced using polypropylene spunbond, polypropylene melt-blown and polyester/rayon spunlace (hydro-entangled) nonwoven layers with the help of a fusing machine and polypropylene powder. Data were measured and statistically analyzed for weight, thickness, bending rigidity, grab tensile properties, bursting strength, air permeability, water vapor transmission rate, hydrostatic pressure, water resistance (impact penetration test), and synthetic fabric blood repellency (unfinished and finished with water repellent solution polymethyl hydrosiloxanes). Because of the growing risk of exposure to blood-borne pathogens such as hepatitis B virus (HBV), hepatitis C virus (HCV), Ebola virus, and human immunodeficiency virus (HIV), surgical gowns have to meet severe requirements. The dual roles of surgical gowns serve as protective barriers against the transfer of micro-organisms and body fluids from the operating staff to the patient and even from the patient to the operating staff. In addition, since they cover a wide area of the body as protective garments, they have a great influence on the wearer's comfort and, ultimately, the effectiveness of the procedure (Rabiee et al. 2020).

\section{Conductive polymers}

Conductive polymers (CPs) are known as a class of organic materials, analogous to inorganic semiconductors and metals, with special electrical and optical properties. Using simple, flexible, and cost-effective approaches, CPs can be synthesized. By using simple electropolymerization methods, they can be conveniently assembled into supra molecular structures with multifunctional capabilities. A number of methodologies have been developed to alter and tune CPs to incorporate and interface them, including biomaterials and biosensors, into biomedical applications. Such new technologies are pursued in a number of biomedical fields, such as bioengineering, regenerative medicine and biosensors, as they could potentially lay the foundations for future breakthroughs (Beyki et al. 2014). Via electrostatic-adsorption and in situ immobilization methods, control-released aromatic-nanocapsules/cotton fabrics with good wash durability and long-lasting fragrance release capability were manufactured without adhesives (Muradi et al. 2018).

\section{Future directions}

In the future, medical textiles will have a growing role. In all additional corporal instruments, external or implanted objects, healthcare and hygienic goods, textiles will, thus, be used. In the production of a variety of medical and surgical 
products, medical textile materials continue to play an important role. In near upcoming 3 to 4 years, the global value of medical textiles market will be like US $\$ 100$ billion. It is very much important to reuse with recycling process with proper sustainability and proper optimization of medical textiles. We need to reuse of medical textiles with recycling process in a healthier condition. Optimum use of medical textiles with maintaining updated eco-friendly technology, establishes a cost-effective and greater economically friendly medical textiles.

\section{Conclusion}

Medical applications rely upon proper antimicrobial execution, necessities for application and anticipated advantages of utilitarian clinical materials. A few significant antimicrobial operators and clinical material improvements are being investigated, including quaternary ammonium salts, silver, and different metals. In the assembling of bio-absorbable filaments for a wide scope of clinical applications, including wound dressings, stitches, nonwoven felts and networks, regular polymers play an indispensable role. They are made of polymer chains cross-connected and are corrupted by the hydrolysis of bonds. Some of the manufacturers are investigating better assembling methods, while others are instructing purchasers on the best way to think about articles of clothing with the goal that they contaminate less. Retailers could assume a significant function in enhancing that message. The $100 \%$ recycling process of medical textile will be a novel dimensional effort for the upcoming future of the world's economy as well as environment.

\section{References}

Abreu MJ et al (2006) 12 - Recycling of textiles used in the operating theatre. In: Wang Y (ed) Recycling in Textiles. Woodhead Publishing, pp 183-202

Ahmad SS et al (2019) The application of recycled Textile and innovative spatial design strategies for a Recycling Centre exhibition space. Procedia Soc Behav Sci 234:525-535

Ahmedet al and C. Ng (2016) Status and challenges of municipal solidwaste management in India: A review. Cogent EnvironmentalScience 2:1139434

Arun J, Gopinath KP (2020) In: Khan A, Inamuddin, Asiri AM (eds) Chemical Recycling of Electronic-Waste for Clean Fuel Production. E-waste Recycling and Management: Present Scenarios and Environmental Issues. Springer International Publishing, Cham, pp 111-126

Beyki MH, Bayat M, Miri S, Shemirani F, Alijani H (2014) Synthesis, characterization, and silver adsorption property of magnetic cellulose xanthate from acidic solution: prepared by one step and biogenic approach. Ind Eng Chem Res 53(39):14904-14912

Brasch et al (2013) Structural Basis for Unique Hierarchical Cylindrites Induced by Ultrahigh Shear Gradient in Single Natural Fiber
Reinforced Poly(lactic acid) Green Composites. Biomacromolecules 15(5):1678-1680

Brasch et al (2014) Temporal solitons in optical microresonators. Nat Photonics 8:145-152

Brasch et al (2015) Photonic-based optical frequency comb using soliton cherenkov radiation. Science aad4811

Brasch J, Rucker M, Haise C (2019) Medical textiles that suit the user: predicting health care workers' preference for disposable versus reusable surgical gowns. Health Mark Q 30(2):162-176

Brennan MB (2018) New age paper and textiles. Chem Eng News Arch 76(12):39-47

Carmean D, Ceze L, Seelig G, Stewart K, Strauss K, Willsey M (2019) DNA data storage and hybrid molecular-electronic computing. Proc IEEE 107(1):63-72

Colquhoun H, Lutz J-F (2014) Information-containing macromolecules. Nat Chem 6(6):455-456

Colquhoun H, Lutz J-F (2021) Information-containing macromolecules. Nat Chem 6(6):455-456

Das O et al (2020) The need for fully bio-based facemasks to counter coronavirus outbreaks: a perspective. Sci Total Environ 736:139611

Dong C, Leber A, Das Gupta T, Chandran R, Volpi M, Qu Y, NguyenDang T, Bartolomei N, Yan W, Sorin F (2020) High-efficiency super-elastic liquid metal based triboelectric fibers and textiles. Nat Commun 11(1):3537

Edwards JV, Vigo TL (2001) Biologically Active Fibers in Health Care, in Bioactive Fibers and Polymers. Am Chem Soc:1-19

Edwards et al (2010) Wildlife friendly oil palm plantations fail to protect biodiversity effectively. Conserv Lett 3(4):236-242

Ergene C, Yasuhara K, Palermo EF (2018) Biomimetic antimicrobial polymers: recent advances in molecular design. Polym Chem 9(18):2407-2427

Grasso M (1995) Recycled textile fibers: The challenge for the twenty-first century. Text Chem Color 27(5):16-20

He L, Li S, Chung CTW, Gao C, Xin JH (2016) Constructing safe and durable antibacterial textile surfaces using a robust graft-to strategy via covalent bond formation. Sci Rep 6(1):36327

He L, Li S, Chung CTW, Gao C, Xin JH (2019) Constructing safe and durable antibacterial textile surfaces using a robust graft-to strategy via covalent bond formation. Sci Rep 6(1):36327

Hou L, Wang L, Wang N, Guo F, Liu J, Chen Y, Liu J, Zhao Y, Jiang L (2016) Separation of organic liquid mixture by flexible nanofibrous membranes with precisely tunable wettability. NPG Asia Mater 8(12):e334-e334

Hou L, Wang L, Wang N, Guo F, Liu J, Chen Y, Liu J, Zhao Y, Jiang L (2019) Separation of organic liquid mixture by flexible nanofibrous membranes with precisely tunable wettability. NPG Asia Mater 8(12):e334-e334

Hug JJ, Krug D, Müller R (2020) Bacteria as genetically programmable producers of bioactive natural products. Nat Rev Chem 4(4):172-193

Karim N, Afroj S, Lloyd K, Oaten LC, Andreeva DV, Carr C, Farmery AD, Kim I-D, Novoselov KS (2020) Sustainable personal protective clothing for healthcare applications: a review. ACS Nano 14(10): 12313-12340

Kozlowski RM, Mackiewicz-Talarczyk M, Barriga-Bedoya J (2010) Natural fibers production, processing, and application: inventory and Future Prospects. Contemp Sci Polym Mater Am Chem Soc 1061:41-51

Kumartasli S, Avinc O (2020) In: Muthu SS, Gardetti MA (eds) Recycling of Marine Litter and Ocean Plastics: A Vital Sustainable Solution for Increasing Ecology and Health Problem. Sustainability in the Textile and Apparel Industries: Sourcing Synthetic and Novel Alternative Raw Materials. Springer International Publishing, Cham, pp 117-137 
Leung CM, Dhand C, Mayandi V, Ramalingam R, Lim FP, Barathi VA et al (2020) Wound healing properties of magnesium mineralized antimicrobial nanofibre dressings containing chondroitin sulphate-a comparison between blend and core-shell nanofibres. Biomater Sci 8(12):3454-3471

Lukasz R et al (2019) Medical textiles with silver/nanosilver and their potential application for the prevention and control of healthcare-associated infections - mini-review. Curr Issues Pharm Med Sci 32(2):104-107

Ma J et al (2020) Green fabrication of control-released, washable, and nonadhesives aromatic-nanocapsules/cotton fabrics via electrostatic-adsorption/in situ immobilization. ACS Sustain Chem Eng 8(40):15258-15267

Martens S, Landuyt A, Espeel P, Devreese B, Dawyndt P, Du Prez F (2018) Multifunctional sequence-defined macromolecules for chemical data storage. Nat Commun 9(1):1-8

Mayandi V, Wen Choong AC, Dhand C, Lim FP, Aung TT, Sriram $\mathrm{H}$ et al (2020) Multifunctional antimicrobial nanofiber dressings containing $\varepsilon$-polylysine for the eradication of bacterial bioburden and promotion of wound healing in critically colonized wounds. ACS Appl Mater Interfaces 12(14):15989-16005

McCullough EA (1993) Methods for determining the barrier efficacy of surgical gowns. Am J Infect Control 21(6):368-374

McCullough ME et al (1995) Forgiveness, feeling connected to others and well being: two longitudinal studies. Pers Soc Psychol Bull 34(2):182-95

Mora M, Wink L, Kögler I, Mahnert A, Rettberg P, Schwendner P, Demets R, Cockell C, Alekhova T, Klingl A, Krause R, Zolotariof A, Alexandrova A, Moissl-Eichinger C (2019) Space Station conditions are selective but do not alter microbial characteristics relevant to human health. Nat Commun 10(1):3990

Moradali MF, Rehm BHA (2020) Bacterial biopolymers: from pathogenesis to advanced materials. Nat Rev Microbiol 18(4):195-210

Moradi F, Ahmadi MS, Mashroteh H (2019) Development of tri-layer breathable fluid barrier nonwoven fabrics for surgical gown applications. J Textile Inst 110(11):1545-1551

Muradi et al (2018) The contribution of agriculture sector in the economy of Afghanistan. Int J Scientific research Management 6(10)

Mutlu H, Lutz J-F (2014) Reading polymers: sequencing of natural and synthetic macromolecules. Angew Chem Int Ed 53(48):13010-13019

Mutlu H, Lutz J-F (2019) Reading polymers: sequencing of natural and synthetic macromolecules. Angew Chem Int Ed 53(48):13010-13019

Newsome C, Newsome A, Newsome N, Newsome V, Stone A (1993) In: Newsome C, Newsome A, Newsome N, Newsome V, Stone A (eds) Alphabetical Listing of Qualifying Organisations Responding to Detailed Questionnaire. European Directory of Hazardous Waste Management 2019/20. Springer Netherlands, Dordrecht, pp $1-420$

Nezakati T et al (2018) Conductive polymers: opportunities and challenges in biomedical applications. Chem Rev 118(14):6766-6843

Overcash M (2012) A comparison of reusable and disposable perioperative textiles: sustainability state-of-the-art 2012. Anesth Analg 114(5):1055-1066

Payne CC (2019) Applications of colloidal silica: past, present, and Future. Colloid Chem Silica Am Chem Soc 234:581-594

Pensupa N et al (2018) Recent Trends in Sustainable Textile Waste Recycling Methods: Current Situation and Future Prospects. In: Lin CSK (ed) Chemistry and Chemical Technologies in Waste Valorization. Springer international publishing, Cham, pp $189-228$

Rabiee N et al (2020) Point-of-use rapid detection of SARS-CoV-2: nanotechnology-enabled solutions for the COVID-19 pandemic. Int J Mol Sci 21:5126
Radu C-D, Parteni O, Ochiuz L (2016) Applications of cyclodextrins in medical textiles - review. J Control Release 224:146-157

Rajan G, Morgan JJ, Murphy C, Torres Alonso E, Wade J, Ott AK, Russo S, Alves H, Craciun MF, Neves AIS (2020) Low operating voltage carbon-graphene hybrid E-textile for temperature sensing. ACS Appl Mater Interfaces 12(26):29861-29867

Rajendran S, Anand SC (2018) Developments in medical textiles. Text Prog 32(4): 1-42

Rajendran C et al (2002) The relationship between service quality and customer satisfaction-a factor specific approach. J Services Marketing 16(4):363-379

Reiter H, Muehlsteff J, Sipilä A (2011) Medical application and clinical validation for reliable and trustworthy physiological monitoring using functional textiles: experience from the HeartCycle and MyHeart project. Annual international conference of the IEEE engineering in medicine and biology society. IEEE engineering in medicine and biology society. Annual International Conference 2011:3270-3273

Rucker MH, Brasch JE, Haise CL (2011) Textile products and prevention of hospital acquired infections: understanding the hospital perspective. J Med Mark 11(2):156-164

Salahudeen N, Rasheed AA (2020) Kinetics and thermodynamics of hydrolysis of crystal violet at ambient and below ambient temperatures. Sci Rep 10(1):21929

Samanta KK, Basak S, Chattopadhyay SK (2015) Recycled fibrous and nonfibrous biomass for value-added Textile and nontextile applications. In: Muthu SS (ed) environmental implications of Recycling and recycled products. Springer Singapore, Singapore, pp 167-212

Sharfman MP, Ellington RT, Meo M (2001) The introduction of postconsumer recycled material into TYVEK ${ }^{\circledR}$ production, marketing, and organizational challenges. J Ind Ecol 5(1):127-146

Shimizu T, Ding W, Kameta N (2020) Soft-matter nanotubes: a platform for diverse functions and applications. Chem Rev 120(4):2347-2407

Sun G (2020) 16 - Antibacterial textile materials for medical applications. In: Pan N, Sun G (eds) Functional Textiles for Improved Performance, Protection and Health. Woodhead Publishing, pp 360-375

Sun M et al (2011) Neuroligin 2 is required for synapse development and funtion at the drosophila neuromuscular junction. J Neuroscience 31(2):687-699

Sungmee P, Jayaraman S (2005) Wearable Sensor Systems: Opportunities and Challenges. in 2020 IEEE Engineering in Medicine and Biology 27th Annual Conference

Swift G (2020) Degradability of commodity plastics and specialty polymers. Agric Synth Polym Am Chem Soc 433:2-12

Takamatsu S, Lonjaret T, Crisp D, Badier J-M, Malliaras GG, Ismailova E (2015) Direct patterning of organic conductors on knitted textiles for long-term electrocardiography. Sci Rep 5(1): 15003

Takamatsu S, Lonjaret T, Crisp D, Badier J-M, Malliaras GG, Ismailova E (2020) Direct patterning of organic conductors on knitted textiles for long-term electrocardiography. Sci Rep 5(1): 15003

Tebyetekerwa M, Xu Z, Yang S, Ramakrishna S (2020) Electrospun nanofibers-based face masks. Advanced Fiber Materials 2(3):161-166

Traka M et al (2019) Association of TAG-1 with Caspr2 is essential for the molecular organization of juxtaparanodal regions of myelinated fibers. J Cell Biol 162(6):1161-1172

Vadicherla T, Saravanan D (2019) Textiles and Apparel Development Using Recycled and Reclaimed Fibers. In: Muthu SS (ed) Roadmap to Sustainable Textiles and Clothing: Eco-friendly Raw Materials, Technologies, and Processing Methods. Springer Singapore, Singapore, pp 139-160 
Wicaksono I, Tucker CI, Sun T, Guerrero CA, Liu C, Woo WM, Pence EJ, Dagdeviren C (2020) A tailored, electronic textile conformable suit for large-scale spatiotemporal physiological sensing in vivo. Npj Flex Electron 4(1):5

Zarybnicka L et al (2017) Application of fluorescent label in polymer nanofibers. Adv Mater Sci Eng 2017:7583245

Zhang T et al (2019) Molecular tagging of a major QTL for fiber strength in upland cotton and its marker-assisted selection. Theor Appl Genet 106(2):262-268

Zhao X, He J-x, Zhan Y-z (2009) Synthesis and characterization of chitosan biguanidine hydrochloride under microwave irradiation. Polym J 41(12):1030-1035

Zhao X, He J-x, Zhan Y-z (2019) Synthesis and characterization of chitosan Biguanidine hydrochloride under microwave irradiation. Polym J 41(12):1030-1035
Zheng J, Suh S (2019) Strategies to reduce the global carbon footprint of plastics. Nat Clim Chang 9(5):374-378

Zhiyuan N, Chen H, Mei H, Zhang T (2014) Molecular tagging of QTLs for fiber quality and yield in the upland cotton cultivar Acala-Prema. Euphytica 195(1):143-156

Zinset al. 2015 Improving waste management in constructionprojects: An Australian study. Resources, Conservation andRecycling 101:73-83

Zins JE, Ellas MJ (2007) Social and Emotional learning: promoting the development of all students. J Educ Psychol Consult 17(2-3):233-255

Zins HM, Howard M (2011) 3 - Reusable medical textiles. In: Bartels VT (ed) Handbook of Medical Textiles. Woodhead Publishing, pp 80-105 